\title{
Ground states of dipolar gases in quasi-1D ring traps
}

\author{
Sascha Zöllner 1 ** \\ ${ }^{1}$ The Niels Bohr International Academy, The Niels Bohr Institute, Blegdamsvej 17, 2100 Copenhagen, Denmark
}

(Dated: December 14, 2011)

\begin{abstract}
We compute the ground state of dipoles in a quasi-one-dimensional ring trap using few-body techniques combined with analytic arguments. The effective interaction between two dipoles depends on their center-of-mass coordinate and can be tuned by varying the angle between dipoles and the plane of the ring. For weak enough interactions, the state resembles a weakly interacting Fermi gas or an (inhomogeneous) Lieb-Liniger gas. A mapping between the Lieb-Liniger and the dipolargas parameters in and beyond the Born approximation is established, and we discuss the effect of inhomogeneities based on a local-density approximation. For strongly repulsive interactions, the system exhibits crystal-like localization of the particles. Their inhomogeneous distribution may be understood in terms of a simple few-body model as well as a local-density approximation. In the case of partially attractive interactions, clustered states form for strong enough coupling, and the dependence of the state on particle number and orientation angle of the dipoles is discussed analytically.

PACS numbers: 67.85.-d, 05.30.Jp, 05.30.Fk
\end{abstract}

\section{INTRODUCTION}

The creation and study of so-called dipolar quantum gases have recently become a major research focus [1, 2]. These gases, such as ultracold atoms with magnetic dipole moments (e.g., Cr $\sqrt[3]{3}$ ) or Dy [4]) or polar molecules (such as KRb [5], LiCs [6] or RbCs [7]), are dominated by dipole-dipole rather than van-der-Waals interactions. Combining a high level of control with long-ranged and strongly anisotropic interactions, dipolar gases offer both the simulation of elusive quantum states in condensedmatter or nuclear physics and the design of exotic novel quantum phases.

The question naturally arises how a dipolar gas behaves when confined to lower dimensions. This is important experimentally, because the lower dimensionality may help alleviate the collisional instability toward headto-tail alignment of the dipoles [8, 9], and conceptually, given that the enhanced quantum fluctuations in lower dimensions give rise to intriguing physics.

In particular, in strictly one dimension (1D), dipolar gases have been shown to exhibit Luttinger-liquid behavior (see, e.g., [10 13]). A finite, quasi-1D transverse confinement may both remove the short-distance divergence of the dipolar interactions [14] and alter the short-range s-wave interactions, which may lead to intriguing physics like a roton instability toward a density wave. In spite of displaying long-range interactions, this system still is an effectively homogeneous $1 \mathrm{D}$ system, so long as the dipoles have a common orientation. In a linear geometry, the anisotropy enters only once the system ceases to be one-dimensional, in which case "zig-zag" chains or other higher-dimensional configurations may show up [15].

*Present address: Helmholtz Center Munich, 85764 Neuherberg, Germany; Electronic address: zoellner@nbi.dk
A dipolar system exhibiting truly $1 \mathrm{D}$ physics as well as anisotropic interactions can be achieved in curved lowerdimensional geometries. Nontrivial geometries are interesting not only in the context of dipolar interactions [16 20], but also for studying, e.g., persistent currents [21, 22]. In the simplest case of a quasi-1D ring trap, which can be realized in the context of cold atoms [2326], the effective 1D dipole interaction between two particles becomes inhomogeneous in the sense that it acquires a dependence on their center-of-mass coordinate. In this paper, we show how by varying both the overall strength as well as the degree of inhomogeneity, interesting regimes are found in such a system - such as a 1D Bose-gas-like phase with center-of-mass dependent interaction $\gamma$, a Wigner crystal with inhomogeneous lattice spacing, and self-bound clusters of identical fermions. These are explained on the basis of numerical few-body calculations as well as by deriving simple analytic models. A short account of these findings has been published recently [27].

This paper is organized as follows. Section II introduces the effective Hamiltonian and discusses aspects of the corresponding two-body problem. In Sec. III, we investigate the many-body ground-state in the limit of homogeneous (repulsive) interactions, i.e., dipoles aligned perpendicular to the plane of the ring. Section IV focuses on the case where the interaction is purely repulsive but inhomogeneous. Partially attractive interactions, as occur for small enough angles between dipoles and the ring plane, are studied in Sec. V] Appendix A contains a concise introduction to the numerically exact multi-configurational time-dependent Hartree method employed for the computation of few-body ground-state properties. 


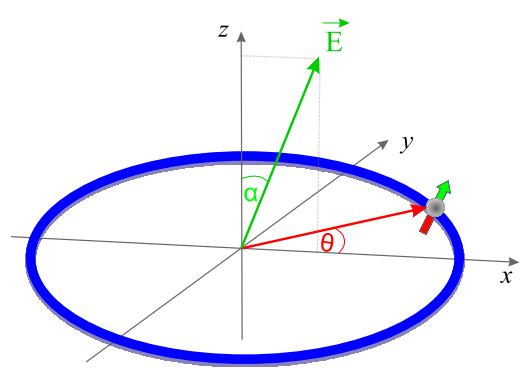

Figure 1: (color online) Sketch of the ring-shaped trap (radius $R$, width $\left.a_{\perp} \ll R\right)$ in the $x y$ plane, with the dipoles moments $\mathbf{d}=d(\sin \alpha, 0, \cos \alpha)^{\top}$ polarized in the $x z$ direction by an external field $\mathbf{E}$.

\section{MODEL}

\section{A. Hamiltonian}

We consider a system of $N$ identical particles (bosons or fermions) of mass $m$, confined in a ring-shaped trapping potential. The ring (radius $R$ ) is taken to lie in the $x y$-plane (Fig. 1). The particles (e.g., atoms with a magnetic moment or polar molecules) have a dipole moment $\mathbf{d}=d(\sin \alpha, 0, \cos \alpha)$ aligned in the $x z$-plane by an external field, at an angle $\alpha$ to the $z$-axis. The interaction between two dipoles in free space is $V(\mathbf{r})=$ $D^{2}\left(1-3 \cos ^{2} \theta_{r d}\right) / r^{3}$, where $\mathbf{r}$ is the separation between the dipoles and $\theta_{r d}$ is the angle between $\mathbf{r}$ and $\mathbf{d}$; furthermore $D^{2}=d^{2} / 4 \pi \epsilon_{0}$ for electric dipoles and $d^{2} \mu_{0} / 4 \pi$ for magnetic ones. Note that for small separations, the far-field dipolar interaction is no longer valid, and shortrange interactions dominate. In order to map out the genuine dipolar physics, we assume that short-ranged forces can be omitted on the length scale of interest. One might imagine tuning them to zero using optical or magnetic Feshbach management; however, it may be important to have short-range repulsion in order to stabilize the dipoles.

We now focus on the limit of a ring potential with tight harmonic confinement in the transverse direction. Then, for sufficiently weak interaction strength, the transverse motion is frozen in the lowest-energy mode, which is a Gaussian $\varphi_{0}(\rho)=e^{-\rho^{2} / 2 a_{\perp}^{2}} / \sqrt{\pi} a_{\perp}$ of spatial extent $a_{\perp} \equiv \sqrt{\hbar / m \omega_{\perp}} \ll R[14]$. Averaging out the transverse degrees of freedom over the reduced density matrix $\hat{\rho}_{\perp}=\left|\varphi_{0}\right\rangle\left\langle\varphi_{0}\right|$, one arrives at an effective 1D Hamiltonian [16]

$$
H=-\frac{\hbar^{2}}{2 m R^{2}} \sum_{i=1}^{N} \frac{\partial^{2}}{\partial \theta_{i}^{2}}+\sum_{i<j} V_{1 \mathrm{D}}\left(\theta_{i}, \theta_{j}\right),
$$

where the angle $\theta_{i}$ specifies the position of particle $i$ on the ring. For $R \gg a_{\perp}$, the effective interaction takes the form $V_{1 \mathrm{D}}\left(\theta_{1}, \theta_{2}\right)=V_{\mathrm{CM}}(\Theta) V_{\text {rel }}(\vartheta)$, where $\Theta=\left(\theta_{1}+\right.$ $\left.\theta_{2}\right) / 2$ is the center-of-mass (CM) angle of the two dipoles and $\vartheta=\theta_{1}-\theta_{2}$ is the relative angle. In terms of $s=$
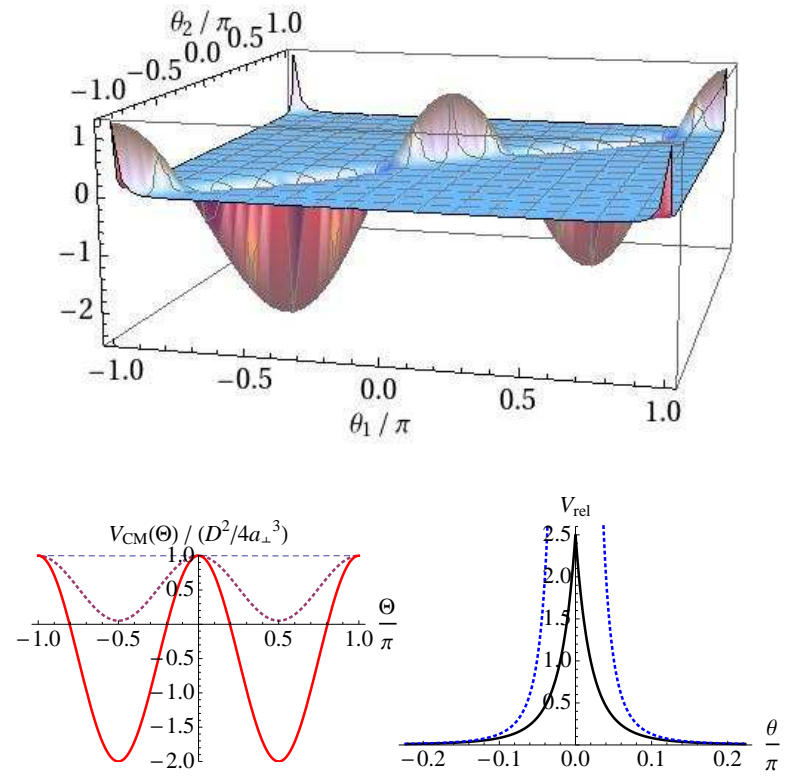

Figure 2: (color online) Effective interaction: (a) Potentialenergy surface $V_{1 \mathrm{D}}\left(\theta_{1}, \theta_{2}\right)$ (here for $\alpha=\frac{\pi}{2}$ ). (b) Center-ofmass-dependent interaction strength $V_{\mathrm{CM}}(\Theta)$ for $\alpha=0,0.19 \pi$ and $\frac{\pi}{2}$. (c) Relative-coordinate potential $V_{\text {rel }}(\vartheta)$ [thick line] for $R / a_{\perp}=10$, including the long-range asymptotics (thin line).

$2 R|\sin (\vartheta / 2)| / a_{\perp}$, the dependence on the relative angle is given by

$$
V_{\text {rel }}(\vartheta)=\sqrt{2 \pi}\left(1+s^{2}\right) e^{s^{2} / 2} \operatorname{erfc}(s / \sqrt{2})-2 s ;
$$

moreover,

$$
V_{\mathrm{CM}}(\Theta)=\frac{D^{2}}{4 a_{\perp}^{3}}\left(1-3 \sin ^{2} \alpha \sin ^{2} \Theta\right) .
$$

Note that the effective interaction $V_{1 \mathrm{D}}$ is inhomogeneous in the angular coordinates: This reflects the anisotropy of the underlying $3 \mathrm{D}$ interaction, which favors configurations where the two dipoles are arranged preferably parallel to d, i.e., such that $\cos \theta_{r d}=\sin \alpha \sin \left(\frac{\theta_{1}+\theta_{2}}{2}\right)$ is maximized, with the particles restricted to the ring. Thus the CM potential, shown in Fig. 2, has minima at $\Theta= \pm \frac{\pi}{2}$, which become more pronounced for larger $\alpha$. For

$$
\alpha>\alpha_{\mathrm{c}} \equiv \arcsin \left(\frac{1}{\sqrt{3}}\right) \approx 0.196 \pi,
$$

the potential acquires attractive regions. The potential should be viewed as a potential-energy surface which depends on both particles' coordinates rather than just the distance between them, as sketched in Fig. 2

The derivation of the effective interaction (2) assumes that the single-mode approximation is valid, i.e., that only the lowest transverse mode $\varphi_{0}$ is occupied. This is 
equivalent to first-order perturbation theory or, in the language of scattering theory, the Born approximation (where $\varphi_{0}(\rho) e^{i q x}$ replaces the $3 \mathrm{D}$ plane-wave states in the presence of confinement). This is valid so long as the interaction energy is small compared with the transverse level spacing, $\hbar \omega_{\perp}$, i.e., if $r_{d} \ll a_{\perp}$. In that case, second-order perturbation theory predicts negative corrections $O\left(\frac{r_{d}}{a_{\perp}}\right)^{2}$ due to virtual excitation of higher transverse levels. For interaction energies of order $\hbar \omega_{\perp}$, confinement-induced resonances may be expected, similar to those for contact interactions under transverse confinement 14, 28].

\section{B. Two-body problem: Relative motion}

Let us briefly discuss some properties of the two-body relative problem, given by the Hamiltonian

$$
h_{\mathrm{rel}}=-\frac{\hbar^{2}}{m R^{2}} \frac{\partial^{2}}{\partial \vartheta^{2}}+\frac{D^{2}}{4 a_{\perp}^{3}} V_{\mathrm{rel}}\left(\frac{r}{a_{\perp}}\right), \quad r \equiv 2 R \sin \frac{\vartheta}{2} .
$$

Strictly speaking, this is relevant only if $\mathrm{CM}$ and relative motions decouple - as in the case of dipoles perpendicular to the plane of the ring $(\alpha=0)$, but also, more generally, for a linear geometry [14]. However, it will also provide useful insight in cases where the particle spacing is small compared with the length scale on which $V_{C M}$ varies, so that one may locally replace $D^{2} / 4 a_{\perp}^{3} \equiv V_{\mathrm{CM}}(\Theta)$ at fixed $\Theta$.

For distances $|r| \gg a_{\perp}$, the free-space dipolar potential is restored, so that the Hamiltonian becomes $a_{\perp}$ independent,

$$
\frac{D^{2}}{4 a_{\perp}^{3}} V_{\mathrm{rel}}\left(\frac{r}{a_{\perp}}\right) \stackrel{|r| \gg a_{\perp}}{D^{2}}
$$

Its strength is characterized by the dipolar length

$$
r_{d} \equiv \frac{2 m}{\hbar^{2}} D^{2}
$$

At short distances, the transverse average introduces a short-range "cutoff" $a_{\perp}$. This attenuates the $r=0$ divergence into a peak of order $D^{2} / a_{\perp}^{3} \cdot[49$

As an illustration, we have diagonalized $h_{\text {rel }}$ numerically and plotted the ground-state wave function $\psi_{\text {rel }}(\vartheta)$ in Fig. 3 for different $r_{d} / R$. For bosons, the noninteracting wave function is the zero-momentum state $\psi(\vartheta)=$ $\frac{1}{\sqrt{2 \pi}}$. For $r_{d} / R \ll 1$, the dominant contribution comes from the peak of $V_{\text {rel }}$ at $\vartheta=0$, where the probability amplitude is reduced. With increasing $r_{d}$, the minimum value of $\psi(0)$ becomes deeper until it reaches down to almost zero (see, e.g., $r_{d} / R=0.4$ ), in which case $\psi(\vartheta) \sim\left|\sin \frac{\vartheta}{2}\right|$ resembles the modulus of the wave function of noninteracting fermions. This behavior is reminiscent of bosons with a short-range (contact) interaction.

For $r_{d} / R \gtrsim 1$, the weaker $|r|^{-3}$ tail becomes effective, driving the two dipoles apart until their density is sharply

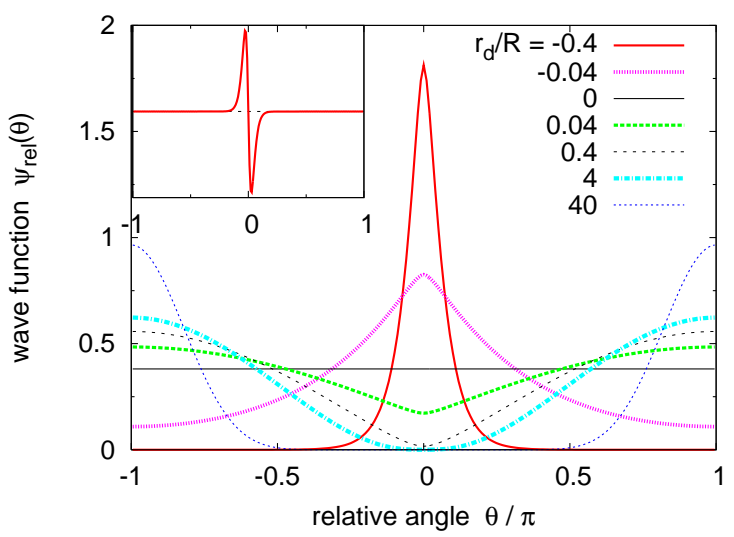

Figure 3: (color online) Relative wave function $\psi(\vartheta)$ of two bosonic dipoles on a ring. For weak repulsive interactions, $r_{d} / R<1$, the $\psi$ resembles that of bosons with contact interactions. For stronger coupling, $r_{d} / R>1$, the wave function becomes localized at $\vartheta= \pm \pi$. For sufficient attraction, the bosons form a bound state. Inset: For identical fermions, a tightly bound state may form for stronger attraction (here $\left.r_{d} / R=-4\right)$.

peaked at a distance $|\vartheta|=\pi$ (i.e, the dipoles are at opposite poles of the ring, $|r|=2 R)$. This is a precursor of a crystal-like state. For fermions, the modulus $|\psi(\vartheta)|$ would look similar.

In the spirit of our comment above, let us consider an effective attraction, $r_{d}<0$. Figure 3 illustrates that two bosons form a bound state, which here becomes localized for $\left|r_{d}\right| / R \gtrsim 0.01$. This is analogous to the wellknown bound state of a $1 \mathrm{D}$ delta interaction. However, even identical fermions can form a "p-wave" bound state (Fig. 3, inset), provided the potential is deep enough to accommodate a second, anti-symmetric level. Note that it owes its existence to the non-zero range $\sim a_{\perp}$ of the potential.

Let us mention that the relative state for $N=2$ fermions (or any even number) on a ring is a subtle issue, as can be seen from the Slater determinant $\left(e^{ \pm i \theta_{2}}-e^{ \pm i \theta_{1}}\right) \propto e^{ \pm i \frac{\theta_{1}+\theta_{2}}{2}} \sin \frac{\theta_{1}-\theta_{2}}{2}$. The CM state has nonzero (angular) momentum \pm 1 , whereas the relative orbital, having momentum $\frac{1}{2}$, is antiperiodic in $\vartheta \equiv \theta_{1}-\theta_{2}$ in order to preserve $2 \pi$-periodicity of the total wave function. This relates to the fact that any even- $N$ Fermi sea is twofold degenerate, and thus will either have nonzero momentum or, in the case of a symmetric Fermi-level state $e^{i k_{F} \theta_{N}}+e^{-i k_{F} \theta_{N}} \propto \cos \left(k_{F} \theta_{N}\right)$, a cosine modulation of the $\mathrm{CM}$ wave function, breaking the translational symmetry.

\section{HOMOGENEOUS CASE $(\alpha=0)$}

Before tackling inhomogeneous interactions, let us first focus on the homogeneous case, $\alpha=0$. Here the dipoles are oriented perpendicular to the plane of the ring; thus, 


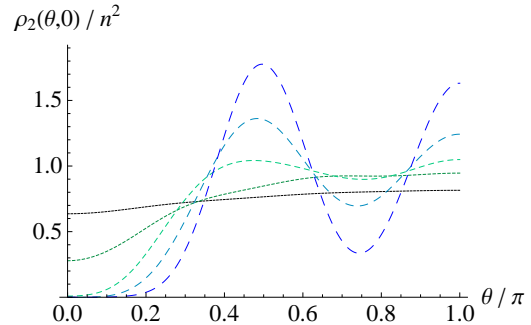

Figure 4: (color online) Pair-distribution function $\rho_{2}(\theta, 0)$ in the homogeneous case $\alpha=0$ (4 bosons), for $n r_{d}=0.0025$, $0.025,0.25,2.5$ and 25.5 (with increasing dashing).

rotational symmetry is preserved and the interaction is independent of $\Theta, V_{1 \mathrm{D}}\left(\theta_{1}, \theta_{2}\right)=D^{2} V_{\text {rel }}\left(\theta_{1}-\theta_{2}\right) / 4 a_{\perp}^{3}$. This system therefore has a close analogy to a linear $1 \mathrm{D}$ system, with particle distances $x_{i}-x_{j} \equiv 2 R \sin \left(\frac{\theta_{1}-\theta_{2}}{2}\right)$. In order to exhibit the essential physics, we first discuss ground-state properties for a few particles as obtained using the numerically exact multi-configurational timedependent Hartree method (see Appendix A). We then proceed to discuss analytical models for the limiting cases of gas-like and solid-like regimes.

\section{A. Ground-state properties}

Since the number density $n(\theta)=\left\langle\sum_{i} \delta\left(\theta-\theta_{i}\right)\right\rangle=$ $N / 2 \pi$ is constant for any $n r_{d}$ due to translational invariance in $\theta$, we focus on two-particle correlations. These are described, e.g., by the pair distribution function $\rho_{2}\left(\theta, \theta^{\prime}\right)=\sum_{i \neq j}\left\langle\delta\left(\theta-\theta_{i}\right) \delta\left(\theta^{\prime}-\theta_{j}\right)\right\rangle$, which gives the probability density of finding one dipole at $\theta$ and a second one at $\theta^{\prime}$. This is plotted in Fig. प for $N=4$ bosons.

For dipole lengths much smaller than the inter-particle distance, $n r_{d} \ll 1$, the initially uniform pair distribution develops a "correlation hole" at $\theta=\theta^{\prime}$ with increasing coupling - indicating that finding two dipoles at the same position becomes more and more unlikely - until the pair distribution strongly resembles that of noninteracting fermions (see, e.g., $n r_{d}=0.25$ ) exhibiting Friedel oscillations. This gas-like behavior is reminiscent of bosons interacting through a short-range or contact interaction $g \delta\left(x-x^{\prime}\right)$ [29], an observation similarly made in the context of a dipolar gas in a harmonic trap [30]. Throughout this regime, fermions would remain essentially noninteracting due to the Pauli principle.

As the dipolar length becomes comparable with the mean inter-particle separation, $n r_{d} \gtrsim 1$, the long-range tail of the interaction becomes crucial. Then the pair distribution develops more pronounced oscillations, which culminate in well-separated peaks for $n r_{d} \gg 1$. These indicate the crystal-like localization of the individual particles [10, 30].

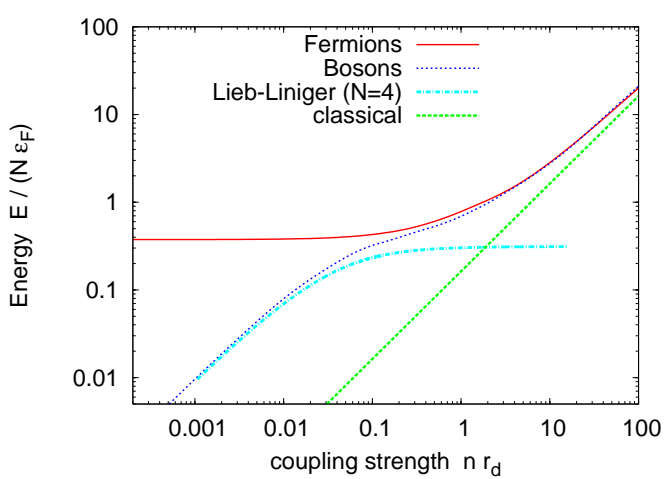

Figure 5: (color online) Ground-state energy $E\left(n r_{d}\right)$ for $N=$ 4 dipoles in the homogeneous system $(\alpha=0)$. Also plotted for comparison: the energy of $N$ Lieb-Liniger bosons (with $\left.g=D^{2} / a_{\perp}^{2}\right)$ and the classical limit $E_{C}$.

\section{B. Bosons: Lieb-Liniger-gas regime $\left(n r_{d} \ll 1\right)$}

For weak dipolar coupling, $r_{d} \ll 1 / n$, we observe a behavior reminiscent of a 1D Bose gas with short-range interactions. Let us now model this gas-like regime. [29]

The 1D Bose gas is described by the Lieb-Liniger model

$$
H_{L L}=-\frac{\hbar^{2}}{2 m} \sum_{i=1}^{N} \frac{\partial^{2}}{\partial x_{i}^{2}}+g \sum_{i<j} \delta\left(x_{i}-x_{j}\right)
$$

where here $x_{i} \equiv R \theta_{i}$. In the thermodynamic limit $N, R \rightarrow \infty$ (keeping the density $n=N / 2 \pi R$ fixed), the properties of the Lieb-Liniger model are completely determined by the dimensionless parameter [29]

$$
\gamma=\frac{m}{\hbar^{2} n} g \equiv-\frac{2}{n a_{1}}>0,
$$

where $a_{1}=-2 \hbar^{2} / m g$ denotes the $1 \mathrm{D}$ scattering length. More specifically, the Lieb-Liniger gas exhibits a crossover from a weakly interacting Bose gas for $\gamma \ll 1$ to a so-called Tonks gas in the "fermionization" limit $\gamma \gg 1$, similar to our findings in the gas-like regime. We will now delineate a relation between the model parameter $\gamma$ (i.e., $g$ ) and the 1D dipolar interaction (parametrized by $\left.r_{d}, a_{\perp}\right)$.

\section{Born approximation for the interaction}

In the spirit of the Born approximation, one may identify the coupling constant with the zeromomentum Fourier transform of the potential, $g \approx$ $V_{1 D}(q=0)=\lim _{q \rightarrow 0} \int d x V_{1 D}(x) e^{i q x}$. For $V_{1 D}(x) \equiv$ $D^{2} V_{\text {rel }}\left(x / a_{\perp}\right) / 4 a_{\perp}^{3}$, this yields

$$
g=\frac{D^{2}}{2 a_{\perp}^{2}} \int_{0}^{\infty} d s V_{\mathrm{rel}}(s)=\frac{D^{2}}{a_{\perp}^{2}} \Longrightarrow \gamma=\frac{r_{d}}{2 n a_{\perp}^{2}} .
$$


To check this, in Fig. [5 we have plotted the groundstate energy $E\left(n r_{d}\right)$ obtained numerically for dipolar interactions as well as for the Lieb-Liniger model. To compare the two on an equal footing, we have made use of the results for $N=4$ bosons based on the solution of the $N$ particle Lieb-Liniger equations [31] (rather than the integral equations valid in the thermodynamic limit), with $g$ given by (4). For $\gamma \ll 1$, i.e., $n r_{d} \ll 2\left(n a_{\perp}\right)^{2} \sim 0.01$, the system is well described by a weakly interacting Bose gas, $E \simeq \frac{N-1}{2} n g$. Conversely, for $2\left(n a_{\perp}\right)^{2} \ll n r_{d} \ll 1$, one has $\gamma \gg 1$, and the bosons repel each other so strongly that they become virtually impenetrable. For very tight confinement, $n a_{\perp} \rightarrow 0$, the bosons are thus practically fermionized already at very low coupling, which is the case for a purely $1 /|r|^{3}$ potential $[10 \mid$.

In the strict limit $g \rightarrow+\infty\left(a_{1} \rightarrow 0^{-}\right)$, the Lieb-Liniger state $\Psi_{a_{1}}$ is closely connected to that of non-interacting identical fermions $\Psi_{F}$ via the so-called Bose-Fermi map [32],

$$
\Psi_{a_{1} \rightarrow 0^{-}}=\mathcal{A} \Psi_{\mathrm{F}} ; \quad \mathcal{A}\left(x_{1} \ldots x_{N}\right) \equiv \prod_{i<j} \operatorname{sgn}\left(x_{i}-x_{j}\right) .
$$

Note that for the ground state, $\Psi_{0}=\left|\Psi_{F}\right|$. Consequently, only phases differ, whereas all local quantities are identical. Thus the pair distribution function (Fig. 4) displays a correlation hole of size $r \sim 1 / k_{F}, k_{F}=\pi n$ being the Fermi wave number, and the energy approaches $E \rightarrow \sum_{|k| \leq k_{F}} \frac{(\hbar k)^{2}}{2 m} \stackrel{N \gg 1}{\simeq} \frac{1}{3} N \epsilon_{F}$, where $\epsilon_{F}=\left(\hbar k_{F}\right)^{2} / 2 m$. Notice, however, that here the fermionization limit is slightly subtle. First, $\gamma$ in (4) cannot tend to infinity so long as $n r_{d} \ll 1$; rather, at $\gamma \gtrsim 1 / 2\left(n a_{\perp}\right)^{2}$, nonzerorange effects come into play which are no longer described by the Lieb-Liniger model. Moreover, for even numbers $N=2,4, \ldots$, the Bose-Fermi map is not applicable on a ring [32], since the Fermi gas has CM momentum $k_{F}=\pi n$ for reasons discussed in Sec. IIB Thus, the Tonks gas has an energy lower than that of the Fermi gas by the CM energy $\left(\hbar k_{F}\right)^{2} / 2 \mathrm{Nm}$. Generally, for any finite $N$, the energy is slightly lower than the thermodynamiclimit result $E=\frac{1}{3} N \epsilon_{F}$ by a factor of $\left(N^{2}-1\right) / N^{2}$.

\section{Beyond the Born approximation}

Let us now discuss the validity of the Born approximation (4) and how to include effects beyond it.

In one dimension, the Born approximation holds for 1D scattering lengths large compared with the scattering wavelength, $\left|k a_{1}\right| \gg 1$, and becomes exact in the non-interacting limit $a_{1} \rightarrow-\infty$. This can be derived straightforwardly from the Born expansion of the T matrix. For low-energy scattering, $k a_{\perp} \ll 1$, this leads to the condition $\left|a_{1}\right| \gg a_{\perp}$. Since for the dipolar interaction we have $\left|a_{1}\right| \simeq a_{\perp}^{2} / r_{d}$, this is equivalent to

$$
r_{d} \ll a_{\perp} .
$$

Thus, the Lieb-Liniger map (4) is valid for $n r_{d} \ll n a_{\perp}$, tightening the constraint found above. As an illustration,

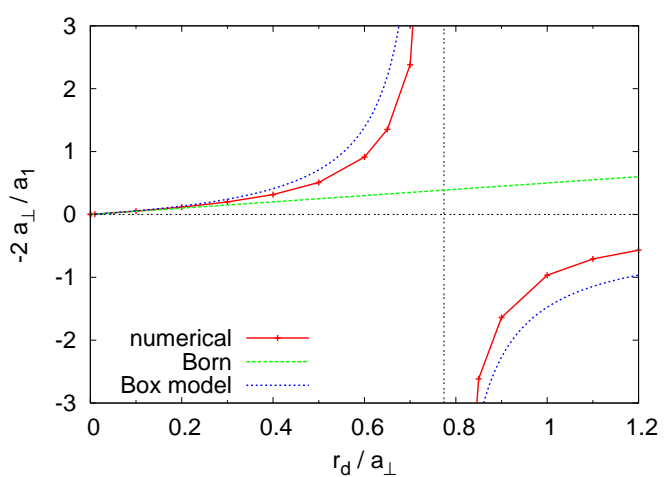

Figure 6: (color online) Effective 1D interaction strength $g \equiv$ $-2 \hbar^{2} / m a_{1}$ (units of $\hbar^{2} / m a_{\perp}$ ) for the dipolar potential $V_{1 D}$ as a function of $r_{d} / a_{\perp}$. For comparison: Born approximation $g \approx D^{2} / a_{\perp}^{2}$, and results for a box potential with corresponding parameters.

for $N=4$ and $R / a_{\perp}=10$, we have $n a_{\perp} \approx 0.064$, i.e., holds up to $\gamma \sim 1 /\left(n a_{\perp}\right)$.

So, what corrections for $g$ do we have to expect for stronger couplings, $r_{d} \gtrsim a_{\perp}$ ? Although that regime is hard to realize experimentally, the question is of theoretical interest. To answer it, we have calculated numerically the $1 \mathrm{D}$ scattering length, $a_{1}=-2 \hbar^{2} / m g$, for the dipolar potential $V_{1 D}$. This is done by solving the $E=0$ Schrödinger equation for the relative motion, $\left[-\frac{\hbar^{2}}{m} \frac{d^{2}}{d r^{2}}+V_{1 D}(r)\right] \psi(r)=0$, and fitting it to the asymptotic low-energy form $\psi(r) \simeq c\left(|r|-a_{1}\right)$ for $|r| \gg a_{\perp}$.

The results are shown in Fig. 6. Clearly, the Born approximation remains qualitatively correct up until $r_{d} / a_{\perp} \approx 0.5$, corresponding to $n r_{d} \approx 0.5 n a_{\perp} \approx 0.032$ above. However, as $r_{d} / a_{\perp} \rightarrow 0.77 \ldots$, a resonance appears, signifying that $g \rightarrow \infty$, and then $g$ crosses over to $g \rightarrow-\infty$. This resonance explains why, in Fig. [5] the fermionization limit was reached already much earlier than naively expected from (4). Moreover, it suggests an interpretation for energies larger than that of the Tonks gas, but still in the gaseous regime: A negative value of $g$ indicates the existence of a so-called Super-Tonks state [33], which exhibits correlations analogous to a hard-core potential with nonzero range $a_{1}>0$. Before discussing this in more depth, let us illustrate briefly why the resonance comes about.

a. Box-potential model To obtain some intuition for interpreting the numerical results obtained above, let us consider a crude model for the dipolar interaction above: a box potential $V(x)=v \Theta(\ell-|x|)$ of range $\ell \sim a_{\perp}$ and height $v \sim D^{2} / a_{\perp}^{3}$. The $1 \mathrm{D}$ scattering length is known analytically,

$$
\frac{a_{1}}{\ell}=1-\frac{\operatorname{coth} \eta}{\eta}, \quad \eta \equiv \sqrt{\frac{m v}{\hbar^{2}}} \ell \sim \sqrt{\frac{r_{d}}{a_{\perp}}} .
$$

Of course, for $\eta \ll 1$, the Born approximation is recovered, $a_{1} / \ell \simeq-1 / \eta^{2}$, or $g \simeq 2 \ell v \sim D^{2} / a_{\perp}^{2}$. However, at $\eta=\eta^{\star} \approx 1.2$ (i.e., for $r_{d}$ of order $a_{\perp}$ ), $a_{1}$ vanishes like 
$a_{1} / \ell \simeq \frac{1}{2}\left(\eta^{2}-\eta^{\star 2}\right)$, so that $g \propto\left(r_{d}-r_{d}^{\star}\right)^{-1}$ exhibits a resonance at the critical point. This is exactly the type of behavior encountered in Fig. 6.

To understand this phenomenon a little more deeply, it is worth considering what happens to the wave function. For $\eta \ll 1$, the (negative) scattering length is much larger than the box radius, $\left|a_{1}\right| \gg \ell$, which reflects that the length scale of the wave function is too large to sample any details of the potential. In other words, the typical energy is so small compared with that associated with the potential's structure, $h^{2} / m a_{1}^{2} \ll \hbar^{2} / m \ell^{2}$, that the physics is basically shape independent and entirely determined by the Born parameter. However, when $\eta \sim 1$, we have $\left|a_{1}\right| \lesssim \ell$, i.e., the essential change occurs within the interior of the box, where the wave function is suppressed more and more strongly. In the extreme case of a hard-sphere potential, $\eta \gg 1, \psi(r)=0$ for all $|r|<\ell$, so that the scattering length $a_{1}=\ell>0$ (in the dipolar case, $a_{1} \rightarrow+\infty$ ). By continuity, $a_{1}$ must change sign in between.

b. Super-Tonks regime The results above permit some analytical statements about the regime where $a_{1}>$ 0 , i.e., beyond the resonance. There the system can be understood as a gas of $1 \mathrm{D}$ hard spheres (rods) with diameter $\ell=a_{1}$. As this resembles a so-called Tonks gas of fermionized bosons, with diameter $a_{1} \rightarrow 0^{-}$, this is sometimes referred to as the Super-Tonks regime [33, 34].

In a homogeneous system, the wave function for $n a_{1}>$ 0 can be obtained from the fermionized wave function $\left(a_{1}=0\right)$ via the mapping [35]

$$
\Psi_{a_{1}}\left(\left\{x_{j}\right\}\right)=\Psi_{0^{-}}\left(\left\{x_{j}-(j-1) a_{1}\right\}\right),
$$

where ordering $x_{j}<x_{j+1}-a_{1}$ is understood, and the total length $L$ entering $\Psi_{0}$ is replaced by $L-N a_{1}$. With that substitution, the hard-sphere energy can be obtained from the fermionized value, $E_{a_{1} \rightarrow 0}=N(\hbar \pi n)^{2} / 6 m$,

$$
E_{a_{1}}=\frac{E_{0}}{\left(1-n a_{1}\right)^{2}} .
$$

In principle, one might expect this formula to also give a good description of the dipolar ground-state energy $E\left(n r_{d}\right)$ when inserting $a_{1}\left(r_{d}\right)$ as obtained from the scattering calculation, at least in the neighborhood of $n a_{1}=0$. However, in our case this regime is not well separated from the long-range one, $n r_{d} \gg 1$, as explained above. Consequently, we do not find Eq. (5) to yield a reasonable agreement for our parameters.

\section{Localization $\left(n r_{d} \gg 1\right)$}

Let us now discuss the limiting case $n r_{\mathrm{d}} \gg 1$. We found that the long-range dipolar repulsion becomes dominant here, so that the particles localize. Another indicator of this is the ground-state energy of the dipoles (Fig. 5), which deviates from the saturated fermionization regime and changes over to an energy increase proportional to $r_{\mathrm{d}}$ for $n r_{d} \gg 1$. In this regime, the dipoles become classical in the sense that the potential energy overwhelms the kinetic energy, so that for $n r_{d} \rightarrow \infty$ the ground state becomes a position eigenstate $\left|\bar{\theta}_{1}, \ldots, \bar{\theta}_{N}\right\rangle_{ \pm}$ ( \pm indicating bosonic/fermionic permutation symmetry), where the angles $\left\{\bar{\theta}_{j}\right\}$ are determined by minimizing the energy

$$
E_{C}=\sum_{i<j} \frac{D^{2}}{\left|2 R \sin \frac{\bar{\theta}_{i}-\bar{\theta}_{j}}{2}\right|^{3}} .
$$

This yields a crystal-like, equidistant distribution of particles: $\bar{\theta}_{j}=j \frac{2 \pi}{N}+\delta, j \in\{0, \ldots, N-1\}$, which is only defined up to an angle $\delta$ by rotational invariance, and an energy $E_{C}=\frac{N D^{2}}{16 R^{3}} \sum_{\nu=1}^{N-1}\left|\sin \frac{\nu \pi}{N}\right|^{-3}$. To find the behavior in the thermodynamic limit, one may approximate the sum by an integral, $\sum_{\nu}\left|\sin \frac{\nu \pi}{N}\right|^{-3} \approx$ $2 \frac{N}{\pi} \int_{\pi / 2 N}^{\pi / 2} d \phi|\sin \phi|^{-3} \simeq 4 N^{3} / \pi^{3}$ as $N \rightarrow \infty$. In that limit, we thus have $E_{C} / N \approx 2 n r_{d} \times(\hbar n)^{2} / 2 m$, an expression formally similar to that of an infinite line [10].

However, for finite $n r_{d}$, the particles are not strictly localized. Rather, their wave packets are spread out over a width $w$ due to their zero-point motion, as is illustrated by the pair distribution function in Fig. 4 We now estimate $w$. For simplicity, we consider a linear 1D system and perform a classical normal-mode analysis: Expanding the interaction potential $D^{2} \sum_{i<j}\left|x_{i}-x_{j}\right|^{-3}$ to second order about the minimum (a lattice with nearest-neighbor distance $1 / n$ ) yields effective harmonicoscillator frequencies $\omega_{C}=\sqrt{12 D^{2} n^{5} / m}$, corresponding to an oscillator width $w \equiv \sqrt{\hbar / m \omega_{C}}$ given by

$$
n w=\sqrt[4]{\frac{1}{12 n m D^{2} / \hbar^{2}}} \sim \frac{1}{\sqrt[4]{n r_{\mathrm{d}}}} .
$$

This confirms that for dipole lengths large compared to the average inter-particle spacing, $n r_{\mathrm{d}} \gg 1$, the dipoles should indeed be well localized, in agreement with Fig. 4. Taking into account the zero-point motion about the classical equilibrium positions, we find that the crystal energy $E_{C}$ is corrected by $+N \hbar \omega_{C} / 2 \sim N(\hbar n)^{2} / m \times$ $\sqrt{n r_{d} / 2}$.

In the crystalline limit $n r_{d} \rightarrow \infty$ where the behavior is nearly classical, the same reasoning applies to fermions. For finite coupling, however, due to the Pauli principle, the kinetic energy of fermions is inherently higher than that of bosons. In this sense, stronger repulsion is needed to suppress the zero-point motion compared to bosons, so that the crystalline limit is approached more slowly.

\section{REPULSIVE INTERACTIONS $\left(0<\alpha<\alpha_{\mathrm{C}}\right)$}

Let us now consider the case where the tilt angle is nonzero such that the effective interaction is still repulsive but inhomogeneous. We first present the groundstate properties obtained for the few-particle system, be- 
fore discussing simple analytic models for the gaseous and crystal-like limits.

\section{A. Ground-state properties}

To bring out the essential effect, we first focus on bosons with an inhomogeneity just below the threshold value, $\alpha=0.19 \pi \lesssim \alpha_{\mathrm{c}}$. In contrast to dipoles aligned perpendicular to the plane of the ring, for $\alpha \neq 0$ the system is no longer rotationally invariant about $\theta$. Thus interaction effects are reflected in the number density $n(\theta)$, as shown for $N=4$ bosons in Fig. 7. For small couplings, $n r_{d} \ll 1$, the state resembles that of a Bose gas. The density has a slightly stronger weight near the potential minima $\theta= \pm \pi / 2$ for intermediate couplings but tends to flatten out for larger values, $n r_{d} \sim 1$. By contrast, for $n r_{d} \gg 1$, the density profile develops $N=4$ distinct peaks, revealing a crystal-like localization of the particles. Note that the peak positions are not equidistant, but rather slightly displaced toward $\theta= \pm \pi / 2$ due to the pronounced minima of $V_{\mathrm{CM}} \cdot 50$.

The transition from a gas-like to a localized state is also clearly visible in the pair distribution function $\rho_{2}\left(\theta, \theta^{\prime}\right)$ shown in Fig. 7? For $n r_{d} \ll 1$, two-body correlations are absent in the mean-field regime, $\rho_{2}\left(\theta_{1}, \theta_{2}\right) \approx \rho\left(\theta_{1}\right) \rho\left(\theta_{2}\right)$, or are limited to Friedel-like oscillations around the correlation hole near $\theta_{1}=\theta_{2}$, reminiscent of a fermionized Bose gas. For $n r_{d} \gg 1$, the pinning of the particles to individual peaks is clearly discernible.

A similar trend would be observed for fermions: For $n r_{d} \ll 1$, we find a gas-like state, which however is only weakly interacting owing to the Pauli exclusion principle. With increasing coupling, $n r_{d} \gtrsim 1$, the state crosses over into a crystal-like one characterized by localization of the particles in the classical minimum-energy configuration, just as in the Bose case. Due to the Fermi energy, that crossover is much smoother than for the bosons.

\section{B. Inhomogeneous Lieb-Liniger regime $\left(n r_{d} \ll 1\right)$}

Let us dwell on the parameter range where the ground state is gas-like. In the homogeneous limit $\alpha \rightarrow 0$, we established that here the system is well described by the Lieb-Liniger Hamiltonian (3), with the 1D interaction strength $g=D^{2} / a_{\perp}^{2}$ (for $r_{d} \ll a_{\perp}$ ). By extension, for $\alpha \neq 0$, the system should resemble a Bose gas with an inhomogeneous interaction $g\left(\frac{x_{1}+x_{2}}{2}\right) \delta\left(x_{1}-x_{2}\right)$, where $g(x)=D^{2}\left(1-3 \sin ^{2} \alpha \sin ^{2} \frac{x}{R}\right) / a_{\perp}^{2}$ is the Fourier transform of $V_{1 D}$ at zero relative momentum.

Although this inhomogeneous Lieb-Liniger Hamiltonian system is not integrable, some insight can be gained by assuming slow variation of $g(x)$ compared with the local correlation length. In this case, a localdensity approximation [36] may be applied, which consists in assuming the equation of state of the homogeneous Lieb-Liniger system, $\mu=f_{g}(n)$, to hold locally,

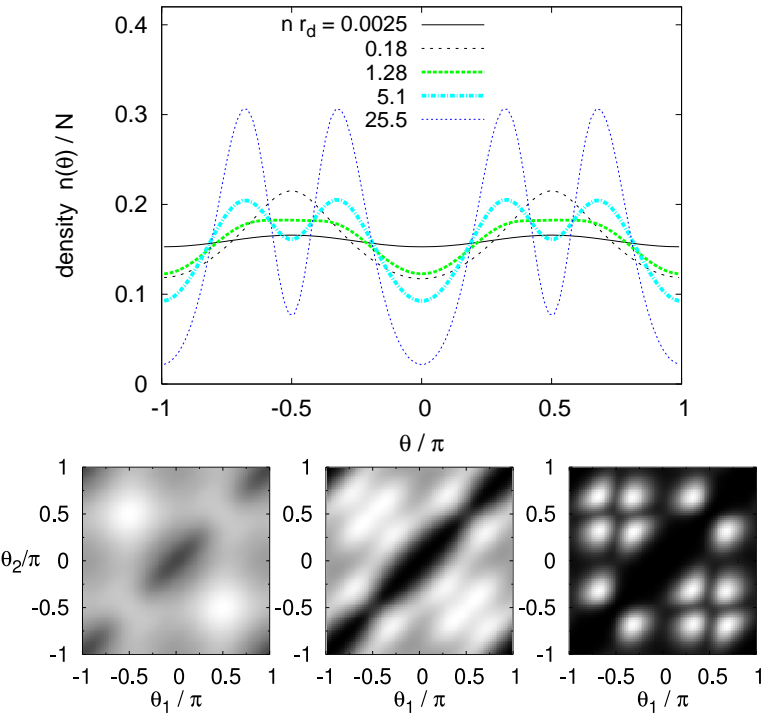

Figure 7: (color online) Inhomogeneous case $(\alpha=0.19 \pi, N=$ 4 bosons): Density profile $n(\theta)$ [top] and pair distribution function $\rho_{2}\left(\theta_{1}, \theta_{2}\right)$ [below] for $n r_{d}=0.025,1.28,25.5$ (left to right).

$\mu=f_{g(x)}[n(x)]$. Here $\mu$ denotes the chemical potential as a function of number density $n(x)$; moreover, for the Lieb-Liniger system, $f_{g}(n)$ can be obtained directly from the ground-state energy, which is known in terms of the dimensionless function $e(\gamma)=E / N \frac{(\hbar n)^{2}}{2 m}$ [29, 37]. Note that the chemical potential is $x$ independent, as local equilibrium is assumed to hold. Carrying out the localdensity approximation numerically for the full range of $\gamma$ is beyond the present scope. However, considering the borderline case also provides some insight into the impact of the inhomogeneity.

\section{1. $\quad$ Mean-field regime, $\gamma(x) \ll 1$}

For locally weak interactions, $\gamma \ll 1$, the homogeneous equation of state reads $\mu=n g$, which yields a profile

$$
n(x)=\frac{\mu}{g(x)}=\frac{n(0)}{1-3 \sin ^{2} \alpha \sin ^{2} \frac{x}{R}} .
$$

Remarkably, this is independent of $\gamma$, and thus of $n r_{d}$, since $\mu \propto g$, that is, kinetic-energy contributions are negligible in the homogeneous equation of state. This holds only for large enough particle numbers $N \gg 1$ and not too large variations of $g(x)$; otherwise the kinetic pressure will smear out the density gradients on the scale of the coherence length or smaller.

It is worth mentioning that the local-density approximation for $\gamma \ll 1$ corresponds to the Thomas-Fermi approximation, which neglects the kinetic energy in the Gross-Pitaevskii equation for the mean-field orbital $\phi(x)$ 
38, Ch. 6],

$$
-\frac{\hbar^{2}}{2 m} \phi^{\prime \prime}(x)=[\mu-n(x) g(x)] \phi(x) \approx 0 .
$$

While the strict mean-field limit assumes all bosons to be condensed into a single orbital, $\Psi=\phi^{\otimes N} / N^{N / 2}$, even for weak repulsion a deviation from a fully uncorrelated state occurs. Conveniently, this can be quantified, e.g., in the second-order correlation function, $g_{2}(x, x) \equiv \rho_{2}(x, x) / n(x)^{2}$. In the homogeneous case, $g_{2}=1-\frac{2}{\pi} \sqrt{\gamma}+O(\gamma)$ [39]. Replacing $\gamma=m g / n \hbar^{2}$ by $\gamma(x)=m g(x, x) / n(x) \hbar^{2} \simeq m g^{2}(x, x) / \hbar^{2} \mu$ yields $g_{2}(x, x) \simeq 1-\frac{2}{\pi} \sqrt{\gamma(0)}\left(1-3 \sin ^{2} \alpha \sin ^{2} \frac{x}{R}\right)$, amounting to a pronounced suppression of the pair distribution $\rho_{2}(\theta, \theta)$ near $\theta \equiv x / R=0$, as observed in Fig. 7 .

\section{Tonks-gas regime, $\gamma(x) \gg 1$}

For stronger coupling, the density modulation (Fig. 7. $\left.n r_{d}=1.3\right)$ is attenuated until, in the fermionization limit $\gamma(x) \gg 1, n(x)$ approaches a constant. This is clear since, by the Pauli principle, identical fermions do not sense any contact interaction and hence no spatial modulation of $g(x)$. Formally, this can be seen from the chemical potential in the Tonks regime, $\mu=(\hbar \pi n)^{2} / 2 m$, which becomes independent of $g$. Since $\mu$ is spatially constant by assumption, so is $n(x)=n(0)$.

For large but finite $g$, pair correlations are actually not fully suppressed, $g_{2} \simeq 0+\frac{4}{3}(\pi / \gamma)^{2}$. Since $\gamma(x) \simeq m g(x) / n \hbar^{2}$, this implies fairly strong residual pair correlations near the potential minima $\theta= \pm \pi / 2$, $g_{2}(x) \simeq \frac{4 \pi^{2}}{3 \gamma(0)^{2}}\left(1-3 \sin ^{2} \alpha \sin ^{2} \frac{x}{R}\right)^{-2}$. Moreover, as discussed for the homogeneous case, the limit $\gamma(x) \gg 1$ corresponds to fermionization only if $n r_{d} \ll 1$, locally. Instead, for increasing $n r_{d}$, we find that the incipient fermionization gives way to a state where the nonzero range of the repulsive potential becomes relevant, until, eventually, a crystal-like localization occurs.

\section{Inhomogeneous crystal-like regime $\left(n r_{d} \gg 1\right)$}

We now present analytical models for the crystal-like limit $n r_{d} \gg 1$. Similarly to the case $\alpha=0$, here each dipole is pinned to a position $\bar{\theta}_{i}$ determined by the extrema of the classical energy

$$
E_{C}=\frac{D^{2}}{8 R^{3}} \sum_{i<j} \frac{1-3 \sin ^{2} \alpha \sin ^{2} \frac{\bar{\theta}_{i}+\bar{\theta}_{j}}{2}}{\sin ^{3}\left|\frac{\bar{\theta}_{i}-\bar{\theta}_{j}}{2}\right|} .
$$

In this case, though, the equilibrium configuration is not simply an equidistant lattice. Rather, owing to the competition of potential energy of the relative motion (favoring maximum distance) and the $\mathrm{CM}$ (alignment near $\theta= \pm \pi / 2)$, the dipoles are localized at discrete positions which are distributed inhomogeneously on the ring. In principle, the distribution of $\left\{\bar{\theta}_{i}\right\}$ can be found by numerical minimization of $E_{C}$. However, to obtain some analytic insight, we will do this for one of the simplest nontrivial cases, $N=4$. Complementing this few-body viewpoint, we then extend the local-density approximation above to the strong-coupling regime.

\section{Few-particle model}

Let us consider the special case $N=4$. By symmetry, we can write $\bar{\theta}_{i}= \pm\left(\frac{\pi}{2} \pm \phi\right), i=1, \ldots, 4$. The onedimensional potential-energy curve

$$
\frac{E_{C}(\phi)}{D^{2} / 4 R^{3}}=\frac{1-3 \sin ^{2} \alpha}{\sin ^{3} \phi}+\frac{1}{\cos ^{3} \phi}+\left(1-3 \sin ^{2} \alpha \sin ^{2} \phi\right)
$$

consists of three contributions:

1. The first term represents the weak repulsion of a pair at, say, $\frac{\pi}{2} \pm \phi$, which has minimum CM energy $(\Theta=\pi / 2)$.

2. The second term represents the energy of a configuration of type $\pm\left(\frac{\pi}{2}+\phi\right)$, which has maximum repulsion $(\Theta=0)$.

3. The remaining term is that of two diametrically opposed dipoles, e.g., at $\pm \frac{\pi}{2}+\phi$; here only the slowly varying $\mathrm{CM}$ potential contributes.

The total potential consists mainly of (1.) a steep potential barrier at $\phi \rightarrow 0$ (repulsion of a pair near $\pm \pi / 2$ ) and (2.) a confinement for larger $\phi$ due to inter-pair repulsion, adding up to a potential well for $\phi$. Neglecting the third term, the potential minimum can be found to be at

$$
\phi_{*}(\alpha)=\arctan \sqrt[5]{1-3 \sin ^{2} \alpha} .
$$

Near $\alpha=0$, this reproduces the uniform lattice spacing $2 \pi / N$, with a very smooth correction for $\alpha>0$, $2 \phi_{*}(\alpha) \simeq \frac{\pi}{2}-\frac{3}{5} \alpha^{2}$. It is only very close to the critical angle that a noticeable inhomogeneity appears, $\phi_{*}(\alpha) \simeq$ $\sqrt[5]{1-3 \sin ^{2} \alpha} \simeq 8 \sqrt[5 / 8]{\sqrt[5]{\alpha_{c}-\alpha}}$. At $\alpha \rightarrow \alpha_{c}^{-}$, this goes to zero very abruptly, indicating an attempted clustering as the minimum energy vanishes. Including the slightly deconfining third term above, the minimum separation angle is marginally shifted toward higher $\phi$. Moreover, although we chose $N=4$ as a special soluble case, the basic competition between bunching toward $\theta= \pm \pi / 2$ and keeping a minimum inter-particle distance will persist for the case of more than a single pair, $N>4$.

Let us comment on the limit $\alpha \rightarrow \alpha_{c}$. The nonanalyticity of $\phi_{*}(\alpha)$ at $\alpha_{c}$ hints at the fact that, at least for $n r_{d} \rightarrow \infty$, there is a phase transition when $\alpha$ crosses the critical angle. In fact, we will see in Sec. $\mathrm{V}$ that for $\alpha>\alpha_{c}$ bound states appear which are tightly localized at $\theta= \pm \pi / 2$ for $n r_{d} \rightarrow \infty$. For $\alpha<\alpha_{c}$, however, the 
two dipoles at, say, $\pi / 2$ cannot get arbitrarily close due to the quantum-mechanical kinetic energy neglected so far, contrary to what we would expect based on (6).

\section{Local-density approximation}

Complementary to the few-particle model discussed above, let us consider the limit $N \rightarrow \infty$. If the CM potential varies on a length scale much larger than the average inter-particle distance, we may assume that, locally, the system behaves similarly to a homogeneous $1 \mathrm{D}$ system. Then the local-density approximation may be applied, i.e., that the equation of state of the homogeneous system, $\mu=f_{r_{d}}(n)$, holds locally, $\mu=f_{r_{d}(\Theta)}[\bar{n}(\Theta)]$. Here $\bar{n}(\Theta)$ is the average number density around $\Theta$, and $r_{d}(\Theta)=r_{d} \times\left(1-3 \sin ^{2} \alpha \sin ^{2} \Theta\right)$.

If we take the chemical potential of the linear homogeneous system for $n r_{d} \rightarrow \infty, \mu=4 \zeta(3) \hbar^{2} n^{3} r_{d} / m$ [10], we find

$$
\bar{n}(\Theta) \propto \frac{1}{\sqrt[3]{1-3 \sin ^{2} \alpha \sin ^{2} \Theta}} .
$$

This density variation is rather smooth due to the strong dipolar repulsion. For smaller $\alpha$, the density is practically homogeneous, with tiny humps near $\Theta= \pm \pi / 2$. For $\alpha \rightarrow \alpha_{c}^{-}$, though, the density becomes markedly peaked and tends toward $\delta\left(\Theta \mp \frac{\pi}{2}\right)$, indicating an instability toward clustering beyond $\alpha_{c}$. Of course, the local-density approximation is valid only for density variations slow compared with the local coherence length: Close to the critical angle, $\bar{n}(\Theta)$ is smeared out on the length scale given by the zero-point motion.

\section{PARTIALLY ATTRACTIVE INTERACTIONS

$$
\left(\alpha_{\mathrm{c}}<\alpha<\alpha_{c 2}\right)
$$

For tilt angles $\alpha>\alpha_{c}$, the interaction potential is no longer purely repulsive but acquires attractive regions for CM angles $|\Theta|=\frac{\pi}{2} \pm \frac{\Delta \Theta}{2}$ (Fig. 21), where

$$
\Delta \Theta=2 \arccos \left(\frac{\sin \alpha_{c}}{\sin \alpha}\right) \equiv \frac{\Delta x}{R} .
$$

Note that for $\alpha \rightarrow \alpha_{c}^{+}$, this attractive segment is vanishingly narrow, $\Delta \Theta \simeq 2^{7 / 4} \sqrt{\alpha-\alpha_{c}}$, whereas for $\alpha \rightarrow \frac{\pi}{2}$, it makes up almost two thirds of the total circumference, $\Delta \Theta / \pi \rightarrow 0.608 \ldots$. With increasing coupling, the competition between repulsion and attraction leads to an intriguing crossover from gas-like states toward clustered "droplets", which we will first discuss on the basis of fewbody simulations, before giving an analytic description of the cluster states.

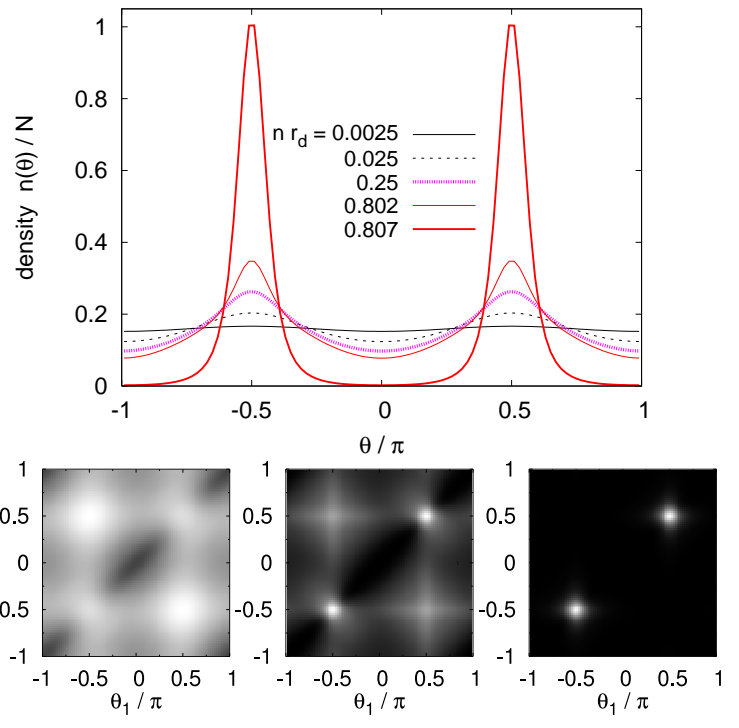

Figure 8: (color online) Clustering of $N=4$ bosons ( $\alpha=$ $0.2 \pi)$ : Density profile $n(\theta)$ [top] and pair distribution function $\rho_{2}\left(\theta_{1}, \theta_{2}\right)$ [below] for $n r_{d}=0.025,0.802$ and 0.807 (left to right).
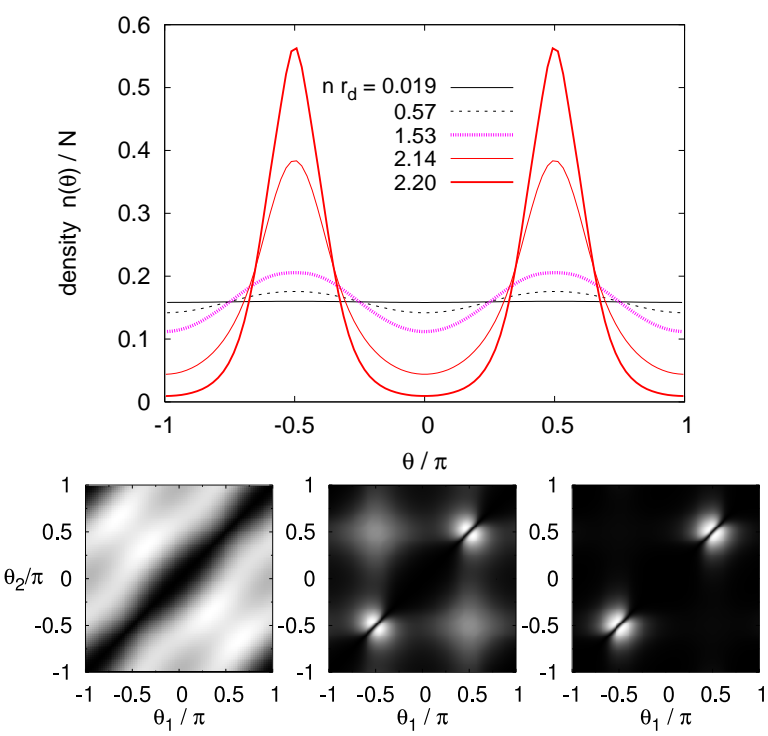

Figure 9: (color online) Clustering of $N=3$ fermions $(\alpha=$ $0.22 \pi)$ : Density profile $n(\theta)$ [top] and pair distribution function $\rho_{2}\left(\theta_{1}, \theta_{2}\right)$ [below] for $n r_{d}=0.57,2.14$ and 2.20 (left to right) .

\section{A. Crossover from gas-like to clustered states}

Let us first illustrate the crossover from weak to strong coupling by means of the density $n(\theta)$, shown in Fig. 8 $(N=4$ bosons, $\alpha=0.2 \pi)$ and Fig. 9 ( $N=3$ fermions, $\alpha=0.22 \pi)$. In both cases, the densities go over from a homogeneous profile, characteristic of a gas-like state, to one with two sharp peaks, indicating cluster formation. 


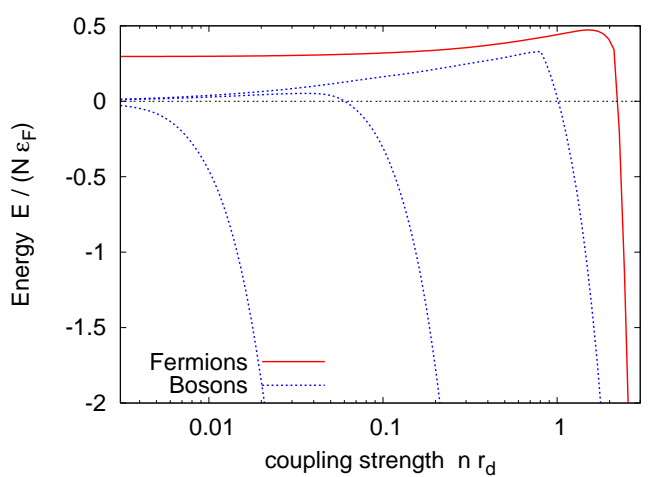

Figure 10: (color online) Ground-state energy $E\left(n r_{d}\right)$ in the case of partial attraction: $N=3$ fermions $(-, \alpha=0.22 \pi)$; $N=4$ bosons $(--, \alpha=0.2 \pi, 0.22 \pi$, and $\pi / 2$ from top to bottom).

It is noteworthy that in these examples, the transition to the localized clustered state is very sharp (e.g., at $n r_{d} \approx 0.8$ for the bosons). This is also reflected in the ground-state energy $E\left(n r_{d}\right)$, shown in Fig. 10. In fact, whereas the energy eventually falls and becomes negative at high enough $n r_{d}$ for any $\alpha>\alpha_{c}$, the behavior for small coupling crucially depends on $\alpha$ : Closer to the critical angle, the bosonic energy increases for small coupling, and it is only beyond a critical value of $n r_{d}$ that it starts decreasing rather abruptly. By contrast, for larger $\alpha, E\left(n r_{d}\right)$ is a monotonically decreasing function. To understand that competition between repulsion and attraction, note that-for $n r_{d} \rightarrow 0$ - the (bosonic) energy behaves as

$$
\begin{aligned}
E & \simeq \frac{N(N-1)}{2}\left\langle V_{\mathrm{CM}}(\Theta)\right\rangle_{0}\left\langle V_{\mathrm{rel}}(\vartheta)\right\rangle_{0} \\
& \sim \frac{N}{2} n g(0)\left(1-\frac{3}{2} \sin ^{2} \alpha\right),
\end{aligned}
$$

where the average is over the (uniform) noninteracting $\mathrm{CM}$ and relative states. Since $g(0)>0$, the energy increase for small $n r_{d}$ is positive for

$$
\alpha<\alpha_{c 2} \equiv \arcsin \sqrt{\frac{2}{3}} \approx 0.304 \pi .
$$

In other words, for tilt angles less than $\alpha_{c}$, the potential is predominantly repulsive. Initially, the delocalized bosons will thus undergo an analogous evolution as in Sec. IV. e.g., behave like an inhomogeneous Bose gas, which is predominantly repulsive $\left(\alpha<\alpha_{c 2}\right)$ or has a net attraction $\left(\alpha>\alpha_{c 2}\right)$. For identical fermions, the interaction-energy shift per particle will be negligible compared with the Fermi energy for all $\alpha$.

This makes clear that the transition to a clustered state also strongly depends on $\alpha$. Beyond $\alpha_{c 2}$, there is a smooth crossover from an attractive gaseous state to a clustered one (Fig. 10) at rather small coupling. By contrast, for only slightly overcrtical $\alpha \in\left(\alpha_{c}, \alpha_{c 2}\right)-$ e.g., $\alpha=0.2 \pi$ in Fig. 8 - the transition takes place at much larger coupling and is more subtle. With increasing $n r_{d}$, the repulsive gas-like state first goes over into a transitional state with strong fragmentation (cf. $\left.n r_{d}=0.802\right)$, which can be thought of roughly as a Mott-insulatortype state $\left|\frac{N}{2}, \frac{N}{2}\right\rangle$ with $N / 2$ dipoles at each of the poles, $\theta= \pm \pi / 2$. This can be best seen from the pair distribution $\rho_{2}\left(\theta, \theta^{\prime}\right)$, Fig. 8 (center), which upon closer inspection confirms that a measurement of one particle at, say, $\pi / 2$ yields a measurement of $\left(\frac{N}{2}-1\right)$ particles at $\pi / 2$ and $\frac{N}{2}$ at $-\pi / 2$. There is a sharp avoided crossing to a clustered state $\left(n r_{d}=0.807\right)$, with all $N$ particles localized at either $\pm \pi / 2$. By parity symmetry, the exact ground state is actually a superposition or Schrödinger-cat state, $\frac{1}{\sqrt{2}}(|N, 0\rangle+|0, N\rangle)$, as is evident from the pair distribution function in Fig. 8, A small symmetry-breaking perturbation will likely cause a collapse of the superposition.

For fermions, the critical interaction strength needed for cluster formation is higher (Fig. 10). This is because of the Pauli principle: The exchange hole discernible in $\rho_{2}$ (Fig. 9) strongly reduces the average attraction between two fermions. Moreover, due to the Pauli pressure, the fermions are more spread out spatially and require a stronger attraction to be squeezed into the narrow zone of attraction.

To obtain a deeper understanding of cluster formation, including its dependence on the particle number and on $\alpha$, we will now develop a simplified analytical model that can reproduce its basic features.

\section{B. Simple cluster model: Homogeneous case}

As a first step, let us consider the case of a homogeneous system with purely attractive interaction. This is interesting in its own right, since clustered states are not limited to a ring geometry. Moreover, as we will see below, this also allows for a qualitative understanding of the inhomogeneous case.

\section{Bosons}

Consider $N$ bosonic dipoles interacting with a homogeneous attractive contact interaction $\bar{g}<0$. (On the ring, we may identify this with the value of $g(x)$ in the minimum $x_{0} / R= \pm \pi / 2, \bar{g}=D^{2}\left(1-3 \sin ^{2} \alpha\right) / a_{\perp}^{2}<0$. Let us assume all bosons to be localized at some $x_{0}$, spread out over a length $L$, e.g., by making a variational ansatz with all particles in a single orbital, $\phi_{L}(x)=$ $e^{-\left(x-x_{0}\right)^{2} / 2 L^{2}} / \sqrt[4]{\pi L^{2}}$. Then, provided $L \gg a_{\perp}$, the interaction energy scales as $E_{\text {int }} \sim N^{2} \bar{g} / L$, whereas $E_{\text {kin }} \sim$ $N \hbar^{2} / m L^{2}$. For $\bar{g}<0$, the total energy $E(L)$ has a local minimum, which yields the size of an equilibrium cluster,

$$
L \sim \frac{\bar{a}_{1}}{N}, \quad \bar{a}_{1} \equiv-\frac{2 \hbar^{2}}{m \bar{g}} .
$$

The characteristic length in the two-body case is, of course, the local 1D scattering length $\bar{a}_{1}>0$, and larger 
$N$ lead to a shrinking of the cluster. The cluster's binding energy is then

$$
E \sim-N^{3} \frac{\hbar^{2}}{m \bar{a}_{1}^{2}} \sim-N^{3} \frac{\hbar^{2}\left|r_{d}\left(\frac{\pi}{2}\right)\right|^{2}}{m a_{\perp}^{4}}
$$

which diverges quadratically with the coupling strength.

Note that in the language of the Lieb-Liniger model, this can be understood as the well-known "bright-soliton" ground state of attractive 1D bosons [40, 41]:

$$
E=-\frac{N\left(N^{2}-1\right)}{6} \frac{\hbar^{2}}{m \bar{a}_{1}^{2}} ; \quad \Psi(\boldsymbol{x}) \propto e^{-\sum_{i<j}\left|x_{i}-x_{j}\right| / \bar{a}_{1}} .
$$

Since $E / N \propto N^{2}$, the binding energy is unbounded in the thermodynamic limit $N \equiv \bar{n} L \rightarrow \infty$, and the state is stable only for a small enough number of particles.

In free space, this cluster size would be extremely large for weak attraction. On the ring, however, for the cluster to form, it must fit into the attractive regions of length $\Delta x=2 R \arccos (1 / \sqrt{3} \sin \alpha)$ around $\theta= \pm \pi / 2$,

$$
L<\Delta x \text {. }
$$

This yields a rough estimate for the critical interaction strength necessary for cluster formation,

$$
n r_{d} \gtrsim \frac{\left(a_{\perp} / R\right)^{2}}{\pi \arccos \left(\frac{1}{\sqrt{3} \sin \alpha}\right)\left|1-3 \sin ^{2} \alpha\right|}
$$

which qualitatively reproduces the parameters necessary for cluster formation found numerically [27].

\section{Fermions}

We now discuss the fermionic case. As above, we assume the fermions to be in an essentially noninteracting state, but with a localization length $L$. Then the kinetic energy, $E_{\text {kin }}=N\left(\hbar k_{F}\right)^{2} / 6 m=N^{3} \pi^{2} \hbar^{2} / 6 m L^{2}$, with $k_{F} \equiv \pi N / L$, has the same structure as in the bosonic case except for a factor of order $N^{2}$ due to the exclusion principle. By contrast, the interaction energy has a more subtle form since the nonzero range of the potential is crucial. Setting again $D^{2}\left(1-3 \sin ^{2} \alpha\right) / a_{\perp}^{2} \equiv \bar{g}<0$ and $\rho_{2}\left(x, x^{\prime}\right)=\left(\frac{N}{L}\right)^{2} g_{2}\left(x-x^{\prime}\right)$, we can write

$$
E_{\mathrm{int}}=\frac{N^{2} \bar{g}}{L} \int_{0}^{L} \frac{d r}{2 a_{\perp}} g_{2}(r) V_{\mathrm{rel}}\left(\frac{r}{a_{\perp}}\right) .
$$

For an ideal Fermi gas,

$$
g_{2}(r)=1-\left[\frac{\sin \left(\frac{\pi N}{L} r\right)}{N \sin \left(\frac{\pi}{L} r\right)}\right]^{2} \stackrel{N \gg 1}{\simeq} 1-\left[\frac{\sin \left(k_{F} r\right)}{k_{F} r}\right]^{2} .
$$

There is no general analytic formula for the integral (11), but we can make statements about limiting cases. First, as $L \ll a_{\perp}$ tends to zero, the integral gives $L V_{\text {rel }}(0) / 2 a_{\perp}$, so

$$
E_{\text {int }} \rightarrow \frac{N^{2} \bar{g}}{2 a_{\perp}} \quad(L \rightarrow 0) .
$$

Being bounded, the interaction energy is thus completely outweighed by the kinetic energy $\left(\propto L^{-2}\right)$, which prevents the collapse toward high-density clusters. On the other hand, for large sizes, $L \gg N a_{\perp}$, the dominant contribution comes from the intermediate zone $a_{\perp} \ll r \ll$ $L / \pi N$ where $V_{\text {rel }}(s) \simeq 4 / s^{3}$ and $g_{2}(r) \simeq \frac{1}{3}\left(k_{F} r\right)^{2}$, and

$$
E_{\mathrm{int}} \sim N^{3} \bar{g} a_{\perp}^{2} \frac{\ln \left(L / \pi N a_{\perp}\right)}{L^{3}} \quad(L \rightarrow \infty) .
$$

This falls off slightly faster with $L$ than the kinetic energy. Hence, fermions cannot form a bound state with arbitrarily large size. This reflects the fact that, for low densities, the attractive interaction energy will be largely canceled by the exchange term. However, binding is possible for an intermediate regime, $a_{\perp} \ll L \ll N a_{\perp}$. Here the density is high enough so that the direct (Hartree) interaction term is large, $g_{2}(r) \approx 1$, but not so high for binding to be suppressed by the kinetic pressure:

$$
E_{\mathrm{int}} \approx \frac{N^{2}}{2 L} \bar{g} \quad\left(a_{\perp} \ll L \ll N a_{\perp}\right) .
$$

It is only for such moderate sizes $L$ that clusters may exist. Under this assumption, the minimum energy is found straightforwardly for

$$
L \approx \frac{\pi^{2}}{3} N \bar{a}_{1}
$$

By the Hartree approximation, this has the same structure as for the Bose cluster, but here the cluster becomes larger for higher particle number $N$ because of the Pauli principle. Note that this is valid only under two constraints:

(i) $L \ll N a_{\perp}$ for the Hartree term to be dominant, i.e., $\bar{a}_{1} \ll a_{\perp}$ or $\left|r_{d}\left(\frac{\pi}{2}\right)\right| \gg a_{\perp}$. Minimizing $E(L)$ numerically, we find the onset of clustering at $\left|r_{d}\left(\frac{\pi}{2}\right)\right| \gtrsim a_{\perp}$, which relaxes the condition.

(ii) $\left|\frac{\partial E}{\partial N}\right| \ll \hbar^{2} / m a_{\perp}^{2}$ for the strictly $1 \mathrm{D}$ description to hold. This is equivalent to $\bar{a}_{1} \gg a_{\perp}$, the complementary condition to the Hartree limit (i). However, since the latter criterion can be relaxed considerably, it is plausible that Fermi clustering may be found in a regime where the physics is no longer strictly $1 \mathrm{D}$. This may require further study, going beyond the bare single-mode 1D description.

The discussion so far assumed a 1D system with a homogeneous attraction. On the ring, the cluster must furthermore fit into the attractive regions, $L<\Delta x$ (9). This yields the estimate

$$
n r_{d} \gtrsim \frac{\frac{2}{3} \pi^{3}\left(n a_{\perp}\right)^{2}}{\arccos \left(\frac{1}{\sqrt{3} \sin \alpha}\right)\left|1-3 \sin ^{2} \alpha\right|}
$$


which essentially differs from the bosonic one (10) by a factor of order $N^{2}$. This turns out to reasonably reproduce the curve $n r_{d}(\alpha)$ obtained from our numerical results (not shown here).

\section{Inhomogeneous case}

Let us now develop a model that goes beyond the intuitive criterion (9) by including the CM dependence of the energy.

\section{Bosons}

So far, in assuming a net attraction $\bar{g}<0$, we have limited ourselves to a cluster state already tightly localized in the potential minimum of $V_{\mathrm{CM}}$. More generally, a CM distribution smeared out about $\Theta= \pm \frac{\pi}{2}$ will shift the average interaction energy upward, possibly even to positive values, i.e., $E_{\text {int }} \sim \frac{N^{2}}{2 L}\langle g(X \equiv R \Theta)\rangle$ with

$$
\langle g(X)\rangle=g\left(R \frac{\pi}{2}\right)+g(0) 3 \sin ^{2} \alpha\left\langle\sin ^{2}\left(\Theta-\frac{\pi}{2}\right)\right\rangle \equiv \bar{g}+\delta g .
$$

For a droplet much smaller than the ring radius, $L \ll$ $R$, we have $\left\langle\sin ^{2}\left(\Theta-\frac{\pi}{2}\right)\right\rangle \simeq \frac{1}{4}\left(\frac{L}{R}\right)^{2}$. This regime applies to a tightly bound cluster with $\alpha \gtrsim \alpha_{c}$ or $n r_{d}$ well above the critical value. Then the total energy has an interaction term $\propto L \times N^{2} g(0) \sin ^{2} \alpha / R^{2}$ corresponding to a linear confinement of the CM, in addition to the attractive term known from the homogeneous case. For convenience, the total energy can be cast in the form

$$
\frac{E}{N \frac{\hbar^{2}}{m L^{2}}} \sim 1-\frac{L N}{\bar{a}_{1}}\left(1-\frac{L^{2}}{\delta x^{2}}\right),
$$

where

$$
\left(\frac{\delta x}{R}\right)^{2} \equiv-\frac{\bar{g}}{\delta g}=4\left(1-\left|\frac{\sin \alpha_{c}}{\sin \alpha}\right|^{2}\right)
$$

happens to give the leading order of the attractive-zone width $\Delta x$ for $\alpha \rightarrow \alpha_{c}$, and differs only marginally even for larger $\alpha$. For $\bar{a}_{1} / N \ll \delta x$, that is, a broad attractive zone which would easily accommodate a cluster with freespace binding length $\bar{a}_{1} / N$, the solution is

$$
L \simeq \frac{\bar{a}_{1}}{N}\left(1-\left|\frac{\bar{a}_{1} / N}{\delta x}\right|^{2}\right) .
$$

To leading order, this recovers a droplet with the freespace binding length, but slightly compressed due to the confining term, repelling the CM from the walls of $V_{\mathrm{CM}}$ as the CM wavepacket spreads out beyond the potential minimum. Conversely, in the case where the free-space cluster would have a size strongly exceeding the attractive region, $\bar{a}_{1} / N \gg \delta x$, the droplet size is indeed much smaller than that:

$$
L \simeq \sqrt[3]{\delta x^{2} \bar{a}_{1} / N}
$$

In this limit, the total energy is clearly positive, and the object is held together by the inhomogeneity - the CM confinement - rather than the attractive mechanism responsible in the homogeneous case.

Note that our above estimate required $L \ll R$, so this puts a bound on how weakly confined the dipoles may be. For larger droplet size $L \gtrsim R$, the CM-confinement term $\delta g$ will become weaker until eventually, as $L \gg R$, it will saturate to $\left\langle\sin ^{2}\left(\Theta-\frac{\pi}{2}\right)\right\rangle \rightarrow \frac{1}{2}$. In that limit, the minimum value $\bar{g}$ will simply be replaced by the uniform average $\langle g\rangle=\bar{g}+g(0) \frac{3}{2} \sin ^{2} \alpha=\left(1-\frac{3}{2} \sin ^{2} \alpha\right) g(0)$. This is exactly equivalent to the result obtained the homogeneous case (7): A bound state can only exist for $\langle g\rangle<0$, i.e., in the parameter regime of average attraction, $\alpha>\alpha_{c 2}$. Its binding length is then given by the analog of (8), with $\bar{a}_{1} \mapsto-2 \hbar^{2} / m\langle g\rangle$ significantly larger due to the weaker binding. Since $L \gtrsim R$ by assumption, this can describe a cluster state with $L<\Delta x$ at the utmost for large $\alpha \approx \pi / 2$.

\section{Fermions}

For fermions, assuming a local-density approximation, the inhomogeneity will have a similar effect, namely, to smear out the position of the cluster about the minimum, so that the average direct-interaction term is increased, $\langle g(X)\rangle=\bar{g}+\delta g$. Under the assumptions made in Sec. $\mathrm{VB}$, this leads to the same results as in the bosonic case but with $\bar{a}_{1} / N$ replaced by $N \bar{a}_{1}$ : For $N \bar{a}_{1} \ll \delta x$, the cluster size $L$ is slightly compressed compared with the homogeneous estimate, $N \bar{a}_{1}$. In the opposite limit, $L \simeq \sqrt[3]{\delta x^{2} N \bar{a}_{1}}$ is somewhat larger than the attractive zone, corresponding to a repulsively bound object. Of course, these estimates are valid only for moderate sizes, $a_{\perp} \ll L \lesssim N a_{\perp}$, as discussed above.

\section{Two-mode description}

After having derived a simple model for the singlecluster state, let us now take a broader perspective on the different phases we found in the regime of strongly inhomogeneous, partially attractive interactions. For simplicity, let us focus on bosons; the fermionic case can be discussed analogously.

- For small enough coupling, the state is always that of a weakly interacting gas. For bosons, this is a Bose gas with net attraction for $\alpha>\alpha_{c 2}$ (net repulsion otherwise), distributed coherently over the regions $x_{ \pm}= \pm \pi R / 2$. If the single-particle orbital occupied by all bosons can be written in terms of two modes localized at $x_{ \pm}, \varphi_{0}(x)=$ $\frac{1}{\sqrt{2}}\left[\varphi_{+}(x)+\varphi_{-}(x)\right]$, each created by the field operator $a_{ \pm}^{\dagger}$, then the many-body ground state takes 
the form

$$
|N\rangle_{0}=\frac{1}{\sqrt{N !}}\left(\frac{a_{+}^{\dagger}+a_{-}^{\dagger}}{\sqrt{2}}\right)^{N}|0\rangle .
$$

- For sufficiently large $n r_{d}$, a cluster state is formed for any $\alpha>\alpha_{c}$. This may be written as a cat state

$$
\frac{1}{\sqrt{2}}(|N, 0\rangle+|0, N\rangle) \equiv \frac{1}{\sqrt{2 N !}}\left(a_{+}^{N}+a_{-}^{N}\right)^{\dagger}|0\rangle .
$$

- Furthermore, for small enough $\alpha \gtrsim \alpha_{c}$, there is a transitional regime for intermediate coupling where the ground state has a Mott-insulator-like character: That is, half of the dipoles are localized near $\theta=+\pi / 2$ and the other half near $-\pi / 2$ (taking $N \in 2 \mathbb{N}$ for simplicity), and the wave function approximately has the structure

$$
\left|\frac{N}{2}, \frac{N}{2}\right\rangle=\frac{1}{\frac{N}{2} !}\left(a_{+}^{\dagger}\right)^{N / 2}\left(a_{-}^{\dagger}\right)^{N / 2}|0\rangle .
$$

Expanding the many-body Hamiltonian $H$ in terms of the two-mode states $\left|N_{+}, N_{-}\right\rangle$, one obtains an effective Hubbard-like Hamiltonian

$$
H_{J, U}=-J \sum_{\left\langle s, s^{\prime}\right\rangle} a_{s}^{\dagger} a_{s^{\prime}}+\frac{U}{2} \sum_{s} \hat{n}_{s}\left(\hat{n}_{s}-1\right),
$$

where $J \equiv\left\langle\varphi_{-}\left|\frac{p^{2}}{2 m}\right| \varphi_{+}\right\rangle$denotes an effective tunnel coupling, $U \equiv\left\langle\varphi_{s}, \varphi_{s}\left|V\left(x, x^{\prime}\right)\right| \varphi_{s}, \varphi_{s}\right\rangle$ is the on-site interaction, and $\hat{n}_{s} \equiv a_{s}^{\dagger} a_{s}(s= \pm)$. As in the derivation of the Hubbard model in the context of external (periodic or double-well) potentials [42], this implicitly assumes tight binding, which requires both tunnel coupling and on-site interaction to be negligible compared with the excitation energy of higher "bands" (i.e., excited states pertaining to the doublet $\left.\varphi_{ \pm}\right)$. Moreover, we have suppressed an overall kinetic-energy offset $\sim N \hbar^{2} / m L^{2}$ and an off-site interaction $U_{ \pm} \hat{n}_{+} \hat{n}_{-}$, falling off as $\left(a_{\perp} / R\right)^{3}$.

It is clear from the considerations in Sec. $\mathrm{VB}$ that the orbitals $\varphi_{ \pm}$(and thus $J, U$ ) are not known a priori but rather depend on $N$ and $D^{2}$. In this sense, diagonalizing the effective Hamiltonian $H_{J, U}$ only yields self-consistent information on the ground state. Nonetheless, mapping the phases of the two-site Bose-Hubbard model [43] to our system may provide some useful insight:

- For weak on-site interaction, $N|U| \ll J$, the ground state is a delocalized superfluid state (14), maximizing the coherence $\left\langle a_{+}^{\dagger} a_{-}\right\rangle$.

- In the limit of strong attraction, $|U| \gg J$, the ground state localizes on a single site, thus maximizing the absolute interaction energy $\sim|U| N^{2} / 2$. By symmetry, the state will be a superposition of all single-site states, corresponding to the cluster state (15).
- For strong repulsion, $N U \gg J$, the energy is dominated by the on-site repulsion. That is minimized by a fragmented or Mott-insulator state (16) with interaction energy $\sim U(N / 2)^{2}$.

This indicates that the transition from a fragmented to a clustered state should take place when $U \sim\langle g(X)\rangle / L$ switches from positive to negative. Clearly, this cannot occur for $\alpha>\alpha_{c 2}$, in which case there is a direct crossover from gas-like to clustered behavior. Near the critical angle in turn, $\alpha_{c} \lesssim \alpha<\alpha_{c 2}, U$ changes from positive to negative at $\bar{a}_{1} / N \lesssim \Delta x$, i.e., for $n r_{d}$ given by the criterion (10). Below that critical coupling, the "repulsively bound" cluster state (see Sec. VC 1) is an excitation on top of the insulator-type state, whereas for higher $n r_{d}$, the attractively bound cluster is the ground state.

\section{CONCLUSION AND OUTLOOK}

In this article, we have shown that dipolar particles confined to a quasi-1D ring exhibit inhomogeneous gas-, solid- and (clustered) droplet-like ground states, due to the underlying anisotropy of the 3D dipole interaction.

The solid-like behavior is essentially independent of the transverse confinement, but differs from that in a linear geometry by a non-equidistant lattice-site distribution whenever the dipoles are inclined toward the plane of the ring $(\alpha \neq 0)$. By contrast, especially the Bose-gaslike states depend crucially on the fact that the transverse confinement length cuts off the $|\mathbf{r}|^{-3}$ divergence of the dipole interaction. This leads to a whole class of states with a tunable interaction parameter, as described by the (inhomogeneous) Lieb-Liniger model, rather than merely a hard-core gas. By the same token, the appearance of attractive interaction regions for dipoles oriented sufficiently close to the plane of the ring leads to bound states clustered around the "poles" of the ring, rather than collapse.

Our numerical results are presented for few particles; however, complementary analytical models support an extrapolation to many-body systems. All results are based on a Born approximation for the effective 1D interaction, i.e., on the occupation of a single transverse mode. An interesting subject of future investigation may thus be how an interaction-induced coupling of several transverse modes affects these results. Moreover, our calculations provide motivation for experimental studies as well as theoretical extensions to dipolar systems in more general curved geometries (including, e.g., coupled quasi$1 \mathrm{D}$ systems [44, 45]).

\section{Acknowledgments}

The author is indebted to C. J. Pethick for many inspiring dicussions and comments on the manuscript. Special thanks also go to M. Girardeau, A. Griesmaier, H.D. Meyer, and N. Zinner. Financial support from the 
German Academy of Sciences Leopoldina (LPDS 200911) is gratefully acknowledged.

\section{Appendix A: Computational method}

We use the numerically exact multi-configurational time-dependent Hartree method [46], a quantumdynamics tool which has been applied to few-body systems of identical bosons as well as mixtures (see, e.g., 47] for details). Its principal idea is to solve the timedependent Schrödinger equation $i \hbar \dot{\Psi}(t)=H \Psi(t)$ as an initial-value problem by expanding the solution in terms of direct (or Hartree) products $\Phi_{j} \equiv \varphi_{j_{1}} \otimes \cdots \otimes \varphi_{j_{N}}$ :

$$
\Psi(\boldsymbol{\theta} ; t)=\sum_{\boldsymbol{j}} A_{\boldsymbol{j}}(t) \Phi_{\boldsymbol{j}}(\boldsymbol{\theta} ; t)
$$

Both coefficients $A_{\boldsymbol{j}}(t)$ and the single-particles basis functions $\varphi_{j}(t)$ are determined from the Dirac-Frenkel variational principle $\left\langle\delta \Psi \mid\left[i \hbar \partial_{t}-H(t)\right] \Psi(t)\right\rangle=0$ [46]. This leads to a coupled system of Schrödinger- and mean-fieldtype equations for $A_{j}$ and $\varphi_{j}$, respectively, which is in- tegrated numerically upon discretization. Note that the expansion coefficients inherit the (bosonic or fermionic) permutation symmetry of the wave function, $A_{P(\boldsymbol{j})}=$ $( \pm 1)^{\operatorname{inv}(P)} A_{\boldsymbol{j}}$. Moreover, in our case of particles on a ring, the single-particle wave functions $\varphi_{j} \in \mathrm{L}^{2}(-\pi, \pi)$ are periodic under translations $\theta \mapsto \theta+2 \pi$, which is ensured by expanding them in an appropriate basis set.

Although designed for time-dependent simulations, it is also possible to apply this approach to stationary states. This is done via relaxation, i.e., by applying the non-unitary imaginary-time evolution operator $e^{-H \tau}$. As $\tau \rightarrow \infty$, this exponentially damps out all contributions as $e^{-\left(E_{m}-E_{0}\right) \tau / \hbar}$ except that from the ground state. In practice, one relies on a more robust scheme termed $\mathrm{im}$ proved relaxation [48]. Here $\langle\Psi|H| \Psi\rangle$ is minimized with respect to both the coefficients $A_{j}$ and the orbitals $\varphi_{j}$. The effective eigenvalue problems thus obtained are then solved iteratively by first solving for $A_{j}$ with fixed orbitals and then 'optimizing' $\varphi_{j}$ by propagating them in imaginary time over a short period. That cycle is then repeated.
[1] M. A. Baranov, Phys. Rep. 464, 71 (2008).

[2] T. Lahaye, C. Menotti, L. Santos, M. Lewenstein, and T. Pfau, Rep. Prog. Phys. 72, 126401 (2009).

[3] T. Lahaye, T. Koch, B. Froehlich, M. Fattori, J. Metz, A. Griesmaier, S. Giovanazzi, and T. Pfau, Nature 448, 672 (2007).

[4] M. Lu, S. H. Youn, and B. L. Lev, Phys. Rev. Lett. 104, 063001 (2010).

[5] S. Ospelkaus, K. Ni, G. Quéméner, B. Neyenhuis, D. Wang, M. de Miranda, J. Bohn, J. Ye, and D. Jin, Phys. Rev. Lett. 104, 30402 (2010).

[6] J. Deiglmayr, A. Grochola, M. Repp, K. Mörtlbauer, C. Glück, J. Lange, O. Dulieu, R. Wester, and M. Weidemüller, Phys. Rev. Lett. 101, 133004 (2008).

[7] J. M. Sage, S. Sainis, T. Bergeman, and D. DeMille, Phys. Rev. Lett. 94, 203001 (2005).

[8] T. Koch, T. Lahaye, J. Metz, B. Fröhlich, A. Griesmaier, and T. Pfau, Nature Phys. 4, 218 (2008).

[9] K. Ni, S. Ospelkaus, D. Wang, G. Quéméner, B. Neyenhuis, M. de Miranda, J. Bohn, J. Ye, and D. Jin, Nature 464, 1324 (2010).

[10] A. S. Arkhipov, G. E. Astrakharchik, A. V. Belikov, and Y. E. Lozovik, JETP Lett. 82, 39 (2005).

[11] R. Citro, E. Orignac, S. De Palo, and M. Chiofalo, Phys. Rev. A 75, 51602(R) (2007).

[12] S. De Palo, E. Orignac, R. Citro, and M. Chiofalo, Phys. Rev. B 77, 212101 (2008).

[13] P. Pedri, S. De Palo, E. Orignac, R. Citro, and M. Chiofalo, Phys. Rev. A 77, 15601 (2008).

[14] S. Sinha and L. Santos, Phys. Rev. Lett. 99, 140406 (2007).

[15] G. Astrakharchik, G. Morigi, G. De Chiara, and J. Boronat, Phys. Rev. A 78, 63622 (2008).

[16] O. Dutta, M. Jääskeläinen, and P. Meystre, Phys. Rev.
A 73, 043610 (2006).

[17] K. T. Law and D. E. Feldman, Phys. Rev. Lett. 101, 096401 (2008).

[18] M. Abad, M. Guilleumas, R. Mayol, M. Pi, and D. M. Jezek, Phys. Rev. A 81, 043619 (2010).

[19] M. Abad, M. Guilleumas, R. Mayol, M. Pi, and D. M. Jezek, Europhys. Lett. 94, 10004 (2011).

[20] M. Maik, P. Buonsante, A. Vezzani, and J. Zakrzewski, arXiv:1107.1103 (2011)

[21] A. Ramanathan, K. C. Wright, S. R. Muniz, M. Zelan, W. T. Hill, C. J. Lobb, K. Helmerson, W. D. Phillips, and G. K. Campbell, Phys. Rev. Lett. 106, 130401 (2011).

[22] L. Amico, A. Osterloh, and F. Cataliotti, Phys. Rev. Lett. 95, 063201 (2005).

[23] S. Gupta, K. W. Murch, K. L. Moore, T. P. Purdy, and D. M. Stamper-Kurn, Phys. Rev. Lett. 95, 143201 (2005).

[24] A. S. Arnold, C. S. Garvie, and E. Riis, Phys. Rev. A 73, 041606 (2006).

[25] W. H. Heathcote, E. Nugent, B. T. Sheard, and C. J. Foot, New J. Phys. 10, 043012 (2008)

[26] K. Henderson, C. Ryu, C. MacCormick, and M. G. Boshier, New J. Phys. 11, 043030 (2009).

[27] S. Zöllner, G. M. Bruun, C. J. Pethick, and S. M. Reimann, Phys. Rev. Lett. 107, 035301 (2011).

[28] M. Olshanii, Phys. Rev. Lett. 81, 938 (1998).

[29] E. H. Lieb and W. Liniger, Phys. Rev. 130, 1605 (1963).

[30] F. Deuretzbacher, J. C. Cremon, and S. M. Reimann, Phys. Rev. A 81, 063616 (2010).

[31] K. Sakmann, A. I. Streltsov, O. E. Alon, and L. S. Cederbaum, Phys. Rev. A 72, 033613 (2005).

[32] M. Girardeau, J. Math. Phys. 1, 516 (1960).

[33] G. E. Astrakharchik, J. Boronat, J. Casulleras, and S. Giorgini, Phys. Rev. Lett. 95, 190407 (2005). 
[34] G. E. Astrakharchik and Y. E. Lozovik, Phys. Rev. A 77, 013404 (2008).

[35] T. Nagamiya, Proc. Phys.-Math. Soc. Jpn 22, 705 (1940).

[36] J. Oliva, Phys. Rev. B 38, 8811 (1988).

[37] V. Dunjko, V. Lorent, and M. Olshanii, Phys. Rev. Lett. 86, 5413 (2001).

[38] C. J. Pethick and H. Smith, Bose-Einstein condensation in dilute gases, 2nd ed. (Cambridge University Press, Cambridge, 2008).

[39] D. M. Gangardt and G. V. Shlyapnikov, Phys. Rev. Lett. 90, 010401 (2003).

[40] J. B. McGuire, J. Math. Phys. 5, 622 (1964).

[41] Y. Castin and C. Herzog, C. R. Acad. Sci., Ser IV 2, 419 (2001).

[42] G. J. Milburn, J. Corney, E. M. Wright, and D. F. Walls, Phys. Rev. A 55, 4318 (1997).

[43] E. J. Mueller, T.-L. Ho, M. Ueda, and G. Baym, Phys. Rev. A 74, 033612 (2006).

[44] B. Wunsch, N. T. Zinner, I. B. Mekhov, S.-J. Huang, D.W. Wang, and E. Demler, Phys. Rev. Lett. 107, 073201
(2011).

[45] M. Dalmonte, P. Zoller, and G. Pupillo, Phys. Rev. Lett. 107, 163202 (2011).

[46] M. H. Beck, A. Jäckle, G. A. Worth, and H.-D. Meyer, Phys. Rep. 324, 1 (2000).

[47] S. Zöllner, H.-D. Meyer, and P. Schmelcher, Phys. Rev. A 74, 053612 (2006).

[48] H.-D. Meyer and G. A. Worth, Theor. Chem. Acc. 109, 251 (2003).

[49] A straightforward way of seeing this is by replacing the transverse Gaussian by a plane wave, $\varphi_{0}(\rho)=\frac{1}{\sqrt{2 \pi a_{\perp}}}$, $\rho<a_{\perp} / 2$ : Then the 1D interaction would have the form $D^{2} /{\sqrt{a_{\perp}^{2}+\rho^{2}}}^{3}$.

[50] For an odd number of dipoles, localization is more subtle. For, say, $N=3$, there are two degenerate ground states classically - one would thus see a symmetry-averaged $2 N=6$ peaks in the density $n(\theta)$, rather just $N=3$. 